\title{
Well test analysis on pressure of viscoelastic polymer solution with variable rheological parameters
}

\author{
Hongjun Yin", Weili Yang, Siyuan Meng, Ming Cai
}

Key Laboratory of Enhanced Oil and Gas Recovery Ministry of Education; Northeast Petroleum University; Daqing, China;

"Corresponding Author: yinhj7176@126.com

Received 27 September 2010; revised 28 October 2010; accepted 2 November 2010.

\begin{abstract}
According to the behavior that the polymer solution has both the characteristics of viscosity and elastic properties, the transient flow mathematical model considered the viscoelasticity of the polymer solution has been established. The model, in which the variation of the rheological parameters during the seepage flow has been also taken into consideration, has been solved using finite-difference method. The type curves have been plotted. The influence of some properties of polymer solution including the viscocity, the elastic properties and the rheological parameters has been analyzed. Compared with the curves of the power-law fluid, it is shown that the pressure derivative curve considering the elasticity of the polymer solution upwarps less at the radial flow regime. Besides, it will come down as the variation of the rheological parameters, which is quite different from the case regarding them as constants. Therefore, in well test analysis on pressure of polymer solution, it's necessary to consider the elasticity and the variation of the rheological parameters.
\end{abstract}

Keywords: Polymer Solution; Viscoelastic; Relaxation Time; Rheological Parameters

\section{INTRODUCTION}

The polymer solution used in the oil field is a typical kind of non-Newtonian fluid and its rheological property in the porous media is very complicated because of the effect of shear degradation, deconcentration, adsorption and entrapment.

\footnotetext{
*Project supported by the National Science Foundation of China (Grant No: 50874023) and by the Heilongjiang Provincial Science and Technology Plan Project (Grant No: GZ09A407) and by the Research Program of Innovation Team of Science and Technology in Enhanced Oil and Gas Recovery (Grant No: 2009td08).
}

Many domestic and foreign scholars have studied its rheological property and established several rheological model. The initial researches on the flow behavior of the non-Newtonian fluid are mainly focus on the power-law fluid. In most well test analysis on pressure of polymer solution, it is also assumed that the polymer solution is pure viscous fluid, only the shear viscosity is considered and the rheological parameters are always treated as constants.

But a large number of experiments have demonstrated that polymer solution has viscoelastic behavior and the rheological parameters changes in the seepage flow process. The viscosity of the polymer solution will decline as the raising of the shear rate at a relatively lower Darcy velocity. In the flow event, the viscosity is the dominant influential factor and the elastic property can be neglected. In addition, the rheological property of polymer solution can be expressed by using pseudo-plastic power-law model. However, once the Darcy velocity exceeds the critical value, the viscosity will raise with the increasing of the shear rate, the elastic effect will enhance gradually and its influence will be too significant to be ignored.

Reference [1] has declared that the effective viscosity of polymer solution is composed of the individual contributions of shear and strain viscosity. The elasticity behavior and rheological property of polymer solution in porous media under the conditions of reservoir flow rate has been studied in [2]. A power-law fluid viscoelastic semi-empirical model which could describe the viscoelastic effect of polymer solution in porous media has been developed in [3]. The researches on the viscoelasticity have promoted the development of percolation theory. Reference [4] has conducted numerical simulation study for improvement of polymer flooding by viscoelastic effect. Reference [5] has studied the flow behavior of viscoelastic fluid, power law fluid and Newtonian fluid in pore throat by numerical method. Difference mathematical models of viscous-elastic polymer solution have been established in [6] and [7] from dif- 
ferent angles. The influence of different factors on the pressure of the formation near the injection well is analyzed too. But the variation of rheological behavior was seldom taken into consideration and the consistency coefficient and the power-law index were always treated as constants. Besides, the researches on the well test of viscoelastic polymer solution were seldom.

Therefore, in this paper, the relationship between elastic viscosity and shear viscosity has been insisted in this paper. In addition, the variation of the rheological parameters in the flow event has been taken into consideration. What's more, the expression of the apparent viscosity of polymer solution which considered the viscoelasticity and the changing parameters of polymer solution has been developed. Then the non-dimensional radial instable flow mathematical model has been established and solved using finite-difference methods. The pressure of different formation points near the bottom of the well at different time has been calculated. Then the well test analysis curves have been plotted. In the end, the influence of different factors on the curves has been analyzed.

\section{APPARENT VISCOCITY OF POLYMER SOLUTION}

\subsection{The Shear Viscosity}

The viscosity of the power-law fluid can be described as:

$$
\mu_{\mathrm{v}}=H(r) r^{n(r)-1}
$$

where $r$ is the radius away from the wellbore, $\mathrm{m} ; \gamma$ is the shear rate, $\mathrm{s}^{-1} ; H(r)$ is the consistency coefficient, $\mathrm{mPa} \cdot \mathrm{s}^{\mathrm{n}} ; n(r)$ is the power-law index of polymer solution, dimensionless.

Reference [8] has studied the variation of rheological behavior through experiments and developed the basic models about the variation of rheological parameters along the seepage flow direction. In this paper it means that the consistency coefficient and the power-law index are changing along the radial direction.

For the well where the polymer solution is injected into, the variation of rheological parameters can be expressed as:

$$
\begin{gathered}
H(r)=H\left(r_{\mathrm{w}}\right) e^{\alpha\left(r-r_{\mathrm{w}}\right)} \\
n(r)=n\left(r_{\mathrm{w}}\right)+\beta\left(r-r_{\mathrm{w}}\right)
\end{gathered}
$$

where $r_{\mathrm{w}}$ is the radius of the wellbore, $\mathrm{m} ; \alpha$ is the variation factor of the consistency coefficient, dimensionless; $\beta$ is the variation factor of the power-law index, dimensionless.
According to the researches in [9], the relationship between the shearing rate and the seeping rate is as follows:

$$
\gamma=\frac{3 n+1}{2 n+1} \frac{v}{\sqrt{2 C^{\prime} K \phi}}
$$

where $v$ is the flow velocity through porous medium, $\mathrm{m} / \mathrm{s}$; $K$ is the permeability of the formation, $\mu^{2} ; c^{\prime}$ is the factor related to the tortuosity of capillary, $2.08 \sim 2.50$; $\Phi$ is the porosity of the formation, dimensionless.

The flow velocity through porous medium can be approximately expressed by using the following linear relation:

$$
v=\frac{q}{2 \pi r h}
$$

where $q$ is the injection rate of the polymer solution, $\mathrm{m}^{3} / \mathrm{d} ; h$ is the reservoir thickness, $\mathrm{m}$.

Then the expression of shearing rate considering the variation of rheological parameters can be derived.

$$
\gamma=\frac{3 n(r)+1}{2 n(r)+1} \cdot \frac{1}{\sqrt{2 C^{\prime} K \phi}} \cdot \frac{q}{2 \pi h} r^{-1}
$$

The power-law index changes with the radius. However, the resulting changes of the shear rate make little sense to the viscosity of the fluid at the same point. As a result, the variation of the power-law index can be ignored when calculating the shearing rate. Then the shearing rate can turn to the expression as follows:

$$
\gamma=\frac{3 n\left(r_{\mathrm{w}}\right)+1}{2 n\left(r_{\mathrm{w}}\right)+1} \cdot \frac{1}{\sqrt{2 C^{\prime} K \phi}} \cdot \frac{q}{2 \pi h} r^{-1}
$$

where $n\left(r_{\mathrm{w}}\right)$ is the power-law index of polymer solution in the bottom hole, dimensionless.

Letting

$$
F_{\mathrm{s}}=\frac{3 n\left(r_{\mathrm{w}}\right)+1}{2 n\left(r_{\mathrm{w}}\right)+1} \frac{1}{\sqrt{2 C^{\prime} K \phi}} \frac{q}{2 \pi h}
$$

The shearing rate can be eventually simplified as:

$$
\gamma=F_{\mathrm{s}} r^{-1}
$$

And the final expression of the shear viscosity of the polymer solution considering the variation of theological parameters can be reached.

\subsection{The Elastic Viscosity}

The relationship between elastic viscosity and shear viscosity has been obtained in [10].

$$
\mu_{\mathrm{e}}=2 \gamma \theta_{\mathrm{f}} \mu_{\mathrm{v}}
$$

where, $\theta_{\mathrm{f}}$ is the relaxation time, $\mathrm{s}$. 


\subsection{The Apparent Viscosity}

There are both the shearing deformation and the elastic deformation when the polymer solution seeping in the porous medium because of the continuous contraction and spreading of the runner. As a result, the apparent viscosity is composed of the shear viscosity $\left(\mu_{\mathrm{v}}\right)$ and the elastic viscosity $\left(\mu_{\mathrm{e}}\right)$.

$$
\mu_{\mathrm{a}}=\mu_{\mathrm{e}}+\mu_{\mathrm{v}}=\left(1+2 \gamma \theta_{\mathrm{f}}\right) \mu_{\mathrm{v}}
$$

According to the expressions of the shear rate and the shear viscosity, the final expression of the apparent viscosity can be obtained.

$$
\begin{aligned}
\mu_{\mathrm{a}}= & \left(1+2 \theta_{\mathrm{f}} F_{\mathrm{s}} r^{-1}\right) H\left(r_{\mathrm{w}}\right) e^{\alpha\left(r-r_{\mathrm{w}}\right)} \\
& F_{\mathrm{s}}^{n\left(r_{\mathrm{w}}\right)+\beta\left(r-r_{\mathrm{w}}\right)-1} r^{1-n\left(r_{\mathrm{w}}\right)-\beta\left(r-r_{\mathrm{w}}\right)}
\end{aligned}
$$

\section{MATHETICAL MODEL AND ITS SOLUTION}

The mathematical model for transient flow of viscoelastic polymer solution is derived with the following equations.

The partial differential equation for transient flow of viscoelastic polymer solution:

$$
\frac{1}{r} \frac{\partial}{\partial r}\left(\frac{r}{\mu_{\mathrm{a}}} \frac{\partial p}{\partial r}\right)=\frac{\phi C_{\mathrm{L}}}{K} \frac{\partial p}{\partial t}
$$

Initial condition:

$$
\left.p\right|_{t=0}=p_{\mathrm{i}}
$$

Inner boundary condition:

$$
-\left.\frac{K}{\mu_{\mathrm{a}}} \frac{\partial p}{\partial r}\right|_{r=r_{\mathrm{w}}}=\frac{q}{2 \pi r_{\mathrm{w}} h}
$$

Outer boundary condition:

$$
\left.\frac{\partial p}{\partial r}\right|_{r=r_{\mathrm{e}}}=0
$$

Define the dimensionless variable as follows:

Dimensionless radius

$$
r_{\mathrm{D}}=\frac{r}{r_{\mathrm{w}}}
$$

Dimensionless pressure

$$
p_{\mathrm{D}}=\frac{2 \pi K h}{q \mu^{*} B} \cdot\left(p-p_{\mathrm{i}}\right)
$$

Dimensionless time

$$
t_{\mathrm{D}}=\frac{K t}{\phi \mu^{*} C_{\mathrm{t}} r_{\mathrm{w}}{ }^{2}}
$$

Where $\mu^{*}$ is the characteristic viscosity, that is the apparent viscosity of the polymer solution at the bottom of the wellbore.

$$
\mu^{*}=\left.\mu_{\mathrm{a}}\right|_{r=r_{\mathrm{w}}}=A r_{\mathrm{w}}{ }^{1-n\left(r_{\mathrm{w}}\right)}+C r_{\mathrm{w}}{ }^{-n\left(r_{\mathrm{w}}\right)}
$$

where

$$
\begin{gathered}
A=H\left(r_{\mathrm{w}}\right) F_{\mathrm{s}}^{n\left(r_{\mathrm{w}}\right)-1} \\
C=2 \theta_{\mathrm{f}} H\left(r_{\mathrm{w}}\right) F_{\mathrm{s}}^{n\left(r_{\mathrm{w}}\right)}
\end{gathered}
$$

According to the dimensionless variable above, the seeping model of the polymer solution considering the variation of the rheological parameters was established:

$$
\left\{\begin{array}{l}
\frac{1}{r_{\mathrm{D}}} \frac{\partial}{\partial r_{\mathrm{D}}}\left(\frac{\mu^{*}}{\mu_{\mathrm{a}}} r_{\mathrm{D}} \frac{\partial p_{\mathrm{D}}}{\partial r_{\mathrm{D}}}\right)=\frac{\partial p_{\mathrm{D}}}{\partial t_{\mathrm{D}}} \\
\left.p_{\mathrm{D}}\right|_{t_{\mathrm{D}}=0}=0 \\
\left.\frac{\mu^{*}}{\mu_{\mathrm{a}}} r_{\mathrm{D}} \frac{\partial p_{\mathrm{D}}}{\partial r_{\mathrm{D}}}\right|_{\mathrm{r}_{\mathrm{D}}=1}=-1 \\
\left.\frac{\partial p_{\mathrm{D}}}{\partial r_{\mathrm{D}}}\right|_{r_{\mathrm{D}}=r_{\mathrm{De}}}=0
\end{array}\right.
$$

The difference equation at the point $(i, j)$ can be established by using Implicit Difference Method.

$$
\begin{aligned}
& a_{i} p_{i-1}^{j+1}+b_{i} p_{i}^{j+1}+c_{i} p_{i+1}^{j+1}=d_{i}(i=1,2, \ldots, N-1) \\
& p_{i}^{0}=0,(i=1,2, \cdots, N-1) \\
& p_{i}-p_{i+1}=d_{0},(i=0) \\
& p_{i}^{j+1}-p_{i-1}^{j+1}=0,(i=N)
\end{aligned}
$$

where

$$
\begin{aligned}
& a_{i}=\frac{\mu_{\mathrm{Di}}}{r_{\mathrm{Di}}^{2} \Delta x^{2}}-\frac{r \mu_{\mathrm{Di}}^{\prime}}{2 r_{\mathrm{Di}}^{2} \Delta x} \\
& b_{i}=\frac{-2 \mu_{\mathrm{Di}}}{r_{\mathrm{Di}}^{2} \Delta x^{2}}-\frac{1}{\Delta t_{j}} \\
& c_{i}=\frac{\mu_{\mathrm{D}}}{r_{\mathrm{D}}{ }^{2} \Delta x^{2}}+\frac{r \mu_{\mathrm{D}}{ }^{\prime}}{2 r_{\mathrm{D}}{ }^{2} \Delta x} \\
& d_{i}=-\frac{1}{\Delta t} p_{\mathrm{Di}}^{j} \\
& d_{0}=\frac{\mathrm{A} r_{w}^{1-n\left(r_{w}\right)} \frac{\left(e^{\Delta x}\right)^{1-n\left(r_{\mathrm{w}}\right)}-1}{1-n\left(r_{\mathrm{w}}\right)}+\mathrm{Cr}_{w}{ }^{-n\left(r_{\mathrm{w}}\right)} \frac{1-\left(e^{\Delta x}\right)^{-n\left(r_{\mathrm{w}}\right)}}{n\left(r_{\mathrm{w}}\right)}}{\mathrm{A} r_{\mathrm{w}}{ }^{1-n\left(r_{\mathrm{w}}\right)}+\mathrm{Cr}_{\mathrm{w}}{ }^{-n\left(r_{\mathrm{w}}\right)}}
\end{aligned}
$$

And $\Delta x=\ln \left(r_{\mathrm{e}} / r_{\mathrm{w}}\right) / \mathrm{N}$, which is the grid spacing; $r_{\mathrm{e}}$ is the radius of the external boundary, $\mathrm{m} ; r_{\mathrm{w}}$ is the radius of the wellbore, $\mathrm{m} ; i$ is the number of the node; $\mathrm{N}$ is the grid number; $p_{\mathrm{e}}$ is the supply boundary pressure, $\mathrm{Pa}$. 
Then the tridiagonal coefficient matrix equation of the dimensionless mathematical model was built up as:

$$
\left[\begin{array}{ccccc}
-1 & 1 & & & \\
a_{1} & b_{1} & c_{1} & & \\
& a_{2} & b_{2} & c_{2} & \\
& & \cdot & \cdot & \cdot \\
& & & -1 & 1
\end{array}\right]\left[\begin{array}{c}
p_{\mathrm{D} 0} \\
p_{\mathrm{D} 1} \\
p_{\mathrm{D} 2} \\
\cdot \\
p_{\mathrm{DN}}
\end{array}\right]=\left[\begin{array}{c}
d_{0} \\
d_{1} \\
d_{2} \\
\cdot \\
p_{\mathrm{e}}
\end{array}\right]
$$

The equation has been solved. The pressure and its derivative value at the bottom of the wellbore at different time have been calculated.

\section{TYPE CURFE OF VISCOELASTIC POLYMER SOLUTION}

According to the solution of the mathematical model, the type curve has been drawn, as may be seen in Figure 1.

As shown in Figure 1, when considering the variation of the rheological parameters and the elasticity of the polymer solution, the characteristic of the type curve is as follows:

The pressure curve and the pressure derivative curve both change along the straight line with the slope of 1 at the pure wellbore storage phase.

In transition section, the pressure curve flattens and the pressure derivative curve appears to be a transportation hump.

The pressure derivative curve goes up after the transition regime. This variation is a comprehensive action of adsorption, shear, and elastic deformation.

The pressure derivative curve goes down once the influence of the changing rheological parameters become obviously.

When the effects of the closed outer boundary play a role, the pressure curve and the pressure derivative curve both go up.

\section{ANALYSIS OF INFLUENTIAL FACTORS}

Some parameters which could influence the characteristics of the type curve have been analyzed. These parameters include the relaxation time, the variation factor of the consistency coefficient and the variation factor of the power-law index, as well as the consistency coefficient and the power-law index of the viscoelastic polymer solutions.

The influence of relaxation time is analyzed on the basis of Figure 2. As the relaxation time increases, the pressure and pressure derivative values increase after the pure wellbore storage phase, the "hump" of the pressure derivative curve in transition section increases and the

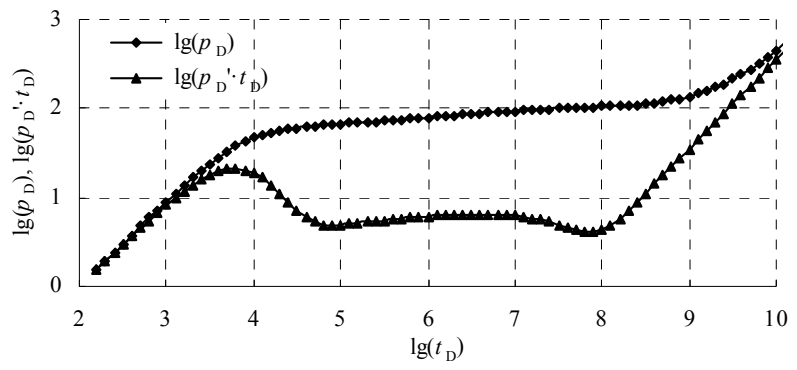

Figure 1. The type curve of viscoelastic polymer solution.

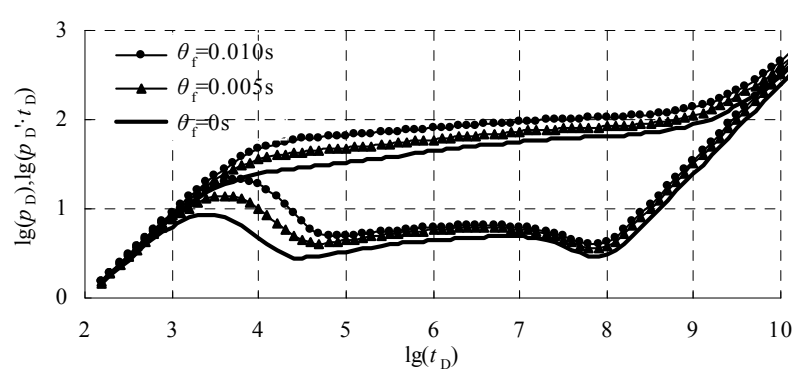

Figure 2. The influence of the relaxation time.

pressure derivative curve upwarps less at the radial flow regime. What's more, compared with the curves simply considered the polymer solution as power-law fluid, it is shown that the pressure derivative curve upturns less at the radial flow regime considering the elasticity of the polymer solution. The larger the relaxation time is, the elastic property of the polymer solution is stronger and the greater the energy is required, then the larger the bottom hole pressure is and the faster it changes. Therefore, it's necessary to consider the elasticity in well test analysis on pressure of viscoelastic polymer solution.

The influence of the consistency coefficient at the wellbore is analyzed on the basis of Figure 3. It is shown that the consistency coefficient of the polymer solution at the wellbore has no significant effect on the go-up of the pressure derivative curve. However, as the consistency coefficient at the wellbore increase, the apparent viscosity of the polymer at the wellbore will increase, the pressure and its derivative value of the radial flow regime will increase.

The influence of the power-law index at the wellbore is analyzed on the basis of Figure 4. The power-law index at the wellbore mainly influences the radial flow regime. As the power-law index at the wellbore decreases, the upturned level of the pressure derivative curve increases. It means that the fluid is Newtonian fluid when the value of the power-law index is 1 . The more the power-law index at the wellbore deviated from the value of 1 , the more obviously of the non-Newtonian flow characteristic is, the greater the flow resistance is and the more obviously the pressure derivative curve goes up. 


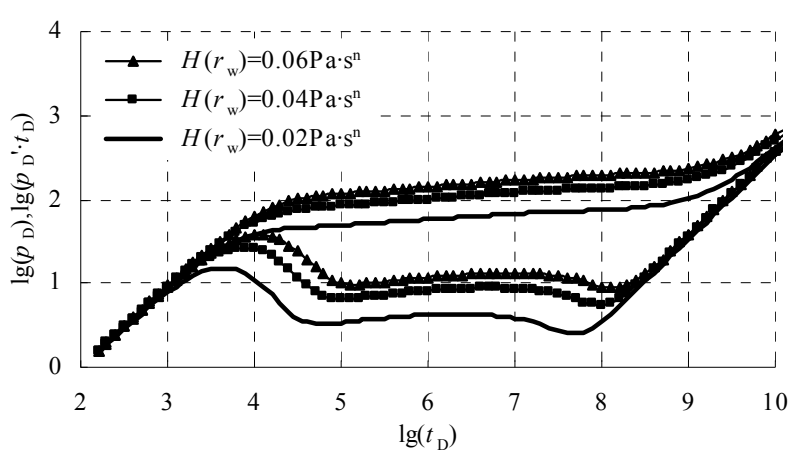

Figure 3. The influence of consistency coefficient at the wellbore.

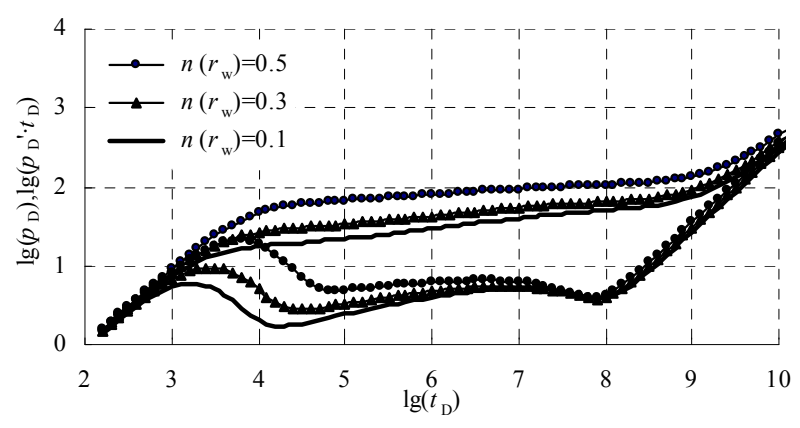

Figure 4. The influence of power-law index at the wellbore.

The influence of variation factor of the consistency coefficient is analyzed on the basis of Figure 5. As the variation factor of the consistency coefficient increases, the radial flow regime disappear earlier, the pressure derivative curve goes down earlier and deeper instead of going on turning up. It is mainly for the following reasons: The smaller the variation factor of the consistency coefficient is, the faster the consistency coefficient changes and the faster the apparent viscosity of polymer solution decreases. In the same period of flowing time, the smaller the apparent viscosity of the polymer solution is, the smaller the flow resistance is. As a result, the pressure derivative curve goes down earlier, the recessed part is wider and the radial flow period stopped earlier.

The influence of variation factor of the power-law index is analyzed on the basis of Figure 6. As the variation factor of the power-law index increases, pressure and pressure derivative values are smaller in the same period of flowing time, the pressure derivative curve upwarps less obviously at the radial flow regime and the radial flow period become shorter. What's more, the Concave appears earlier and deeper. It is mainly for the following reasons. The greater the variation factor of the power-law index is, the faster the power-law index changes, the sooner the apparent viscosity of polymer solution decreases, then the smaller the apparent viscosity is and the lower the flow resistance is. It is the reasons

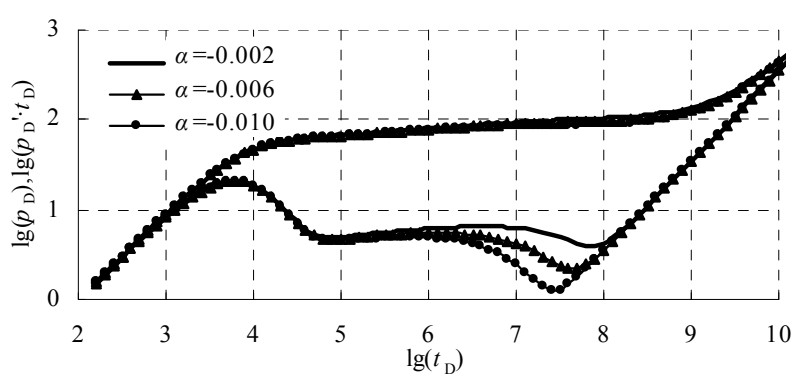

Figure 5. The influence of the variation factor of consistency coefficient.

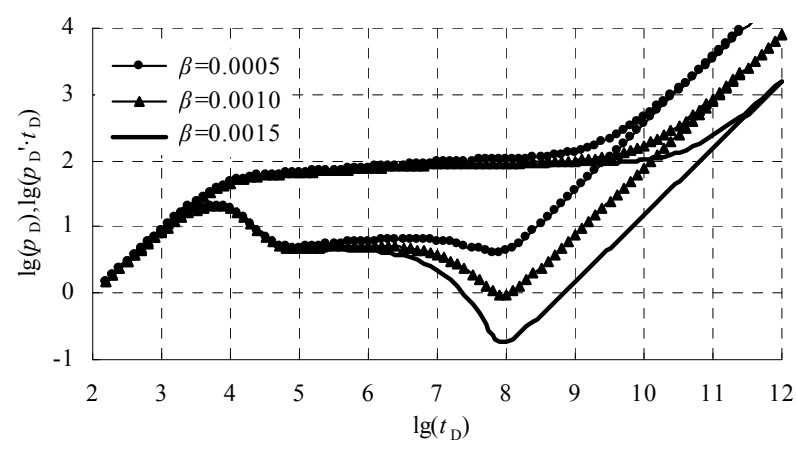

Figure 6. The influence of variation factor of the power-law index.

above make the pressure derivative curve falls earlier.

\section{CONCLUSIONS}

According to the behavior that the polymer solution has viscoelasticity and its rheological parameters are not constants along the seepage flow direction, the transient flow mathematical model has been established. It has been found that the type curve is different from the curves without considering the viscoelasticity and the rheological parameters' variation.

Compared with the curves simply considered the polymer solution as power-law fluid, the pressure curve of viscoelastic polymer solution is higher and the pressure derivative curve upturns less in the radial flow regime.

The greater the relaxation time is, the greater the elastic viscosity of polymer solution is and then the greater the seepage resistance the fluid encountered is. It means that the energy required in the seepage flow is higher. So the injection pressure required is higher and the pressure at the other point of the formation is higher.

The smaller the power-law index of polymer solution in the bottom hole is, the more seriously the non-Newtonian behavior is. Hence, at the same rate, the apparent viscosity of the polymer solution near the bottom hole is smaller. This will make the pressure changes more slow- 
ly and make the pressure derivative curve upturns more at the radial flow regime. The consistency coefficient of polymer solution in the bottom hole has no significant influence on the upturned degree of the pressure derivative curve. It mainly has impact on the value of the pressure and the pressure derivative. The larger the consistency coefficient of polymer solution in the bottom hole is, the larger the value of the pressure and the pressure derivative is.

Because of the variation of the rheological parameters, the apparent viscosity of polymer solution reduces continuously in the flow process, which makes the pressure derivative curve declines after the upturned section in the radial flow and make a concave section appears before the upturned section caused by the closed outer boundary. The more seriously the rheological parameters changes, the more obviously the concave will be.

\section{REFERENCES}

[1] Ranjbar, M., Rupp, J., Pusch, G. and Meyn, R. (1992) Quantification and optimization of viscoelastic effects of polymer solutions for enhanced oil recovery. Society of Plastics Engineers, 24154, 521-531.

[2] Wang, W.Y. (1994) Viscoelasticity and rheological property of polymer solution in porous media. Journal of $\mathrm{J} i$ anghan Petroleum Institute, 16, 54-57 (in Chinese).

[3] Zhang, Y.L., Li, C.H., Wang, X.M. and Hu, J.B. (1994)
The viscoelastic effects of polymer solutions in porous media. Journal of Daqing Petroleum Institute, 18, 139143 (in Chinese).

[4] Li, H., Cheng L.S. and Zhang S.Y. (2002) Numerical simulation study for improvement of polymer flooding by viscoelastic effect. Petroleum Exploration and Development, 29, 91-93 (in Chinese).

[5] Zhang, L.J., Yue, X.A., Liu, Z.C. and Hou, J.R. (2005) Percolation mechanism of polymer solution through porous media. Journal of University Petroleum (Natural Science Edition), 29, 51-55 (in Chinese).

[6] Yin, H.J., Fu, C.Q. and Lv, Y.P. (2004) An unsteady seepage flow model of viscoelastic polymer solution. Journal of Hydrodynamics, 16, 209-215 (in English)

[7] Cao, R.Y., Cheng, L.S., Hao B.Y., Gao, H.H. and Yao, D.W. (2007) Mathematical model of viscous-elastic polymer solution seepage. Journal of Xi' an Shiyou University (Natural Science Edition), 22, 107-109 (in Chinese).

[8] Xia, H.F., Yue, X.A., Cao, G.S. and Zhang, S.F. (2000) Rheological behavior of polymer solution in the course of seepage flow. Journal of Daqing Petroleum Institute, 24, 26-29 (in Chinese).

[9] Wang, X.H. and Zhao, G.P. (1998) Shear rate of powerlaw fluid through porous media. Xinjiang Petroleum Geology, 19, 312-314 (in Chinese).

[10] Xia, H.F. (2002) The theory and application of polymer solution with visco elastic behavior. Petroleum Industry Press, Beijing, 97-101 (in Chinese). 